\title{
Rotating full Poincaré beams
}

\author{
Krasnoshchekov Ye. A., Yaparov V. V. and Taranenko V. B. \\ International Center "Institute of Applied Optics", National Academy of Sciences of \\ Ukraine, 10-G Kudryavska Street, 04053 Kyiv, Ukraine, \\ E-mail: evgen_krasnoshokov@meta.ua
}

Received: 09.11 .2016

\begin{abstract}
We demonstrate numerically the existence and dynamical properties of rotating full Poincaré beams. They are generated either spontaneously or by injection of an optical field into the isotropic laser containing no selective elements except for a circular diaphragm that controls the Fresnel number of laser cavity. For moderate Fresnel numbers, five different kinds of full Poincaré laser modes are available. Each of these modes has an axially symmetric total intensity profile and a distinct polarization pattern that rotates around the beam axis with a constant velocity.
\end{abstract}

Keywords: full Poincaré beams, vector laser dynamics, polarization multistability

PACS: 47.54.-r, 42.60.Jf, 42.25.Ja

UDC: $621.373 .826+535.41$

\section{Introduction}

Recently, vector beams with transversely inhomogeneous polarization structures, also known as Poincaré beams, have drawn much attention of researchers due to their unusual properties which are attractive for potential applications. Well known examples of such beams are cylindrical vector beams [1] that can be focused tighter than comparable scalar beams with a fixed (homogeneous) polarization. The state of linear polarization for these beams changes azimuthally or radially, so that the projection points of the Stokes parameters span the equator of the Poincare sphere. If the polarization state depends on both azimuthal and radial coordinates so that the projections of the Stokes parameters cover the entire Poincaré sphere, then all possible polarization states are present in the beam cross section. Such vector beams are termed as full Poincaré beams [2]. They carry nonzero angular momenta and can be used, e.g., for manipulating submicron-size particles [3].

The vector beams are usually created using active or passive anisotropic optical systems. So, the polarization-mode selection can be provided by means of axial birefringent or dicroic optical elements placed inside a laser cavity, which force the laser to generate azimuthally or radially polarized cylindrical vector beams [1]. These vector beams, together with the higher-order polarization states of generalized vector vortex beams described by the higher-order Poincaré sphere [4], can be generated by a laser with controllable anisotropy. This can be implemented while changing the angle between the principal axes of intracavity quarter-wave plate and q-plate [5]. In the passive schemes, two Laguerre-Gauss modes are collinearly superimposed in a polarization interferometer to form different types of the vector beams [6]. For example, the firstorder full Poincaré beam can be created as a result of coaxial superposition of the fundamental $\mathrm{TEM}_{00}$ mode and the spiral-phase mode $\mathrm{TEM}_{01 *}$, which have orthogonal polarizations. A common feature of different kinds of the Poincaré beams created using these and some other known anisotropic optical systems is that their polarization structure in a fixed transverse plane does not change in time. In other terms, these vector beams remain static.

Ukr. J. Phys. Opt. 2017, Volume 18, Issue 1 
In this study we demonstrate numerically, within the frame of the vector laser model, the existence and dynamical properties of permanently rotating full Poincaré beams (abbreviated as RFP-beams). We prove that they can be generated either spontaneously or by injecting an optical field in an isotropic laser which contains no selective elements except for a circular diaphragm. The diaphragm is used for changing the Fresnel number $N_{F}$ of the cavity $\left(N_{F}=r^{2} / \lambda L\right.$, with $r$ being the radius of the diaphragm, $\lambda$ the wavelength of laser radiation and $L$ the laser cavity length).

\section{Vector laser model}

We consider a transversely multi-mode and longitudinally single-mode laser with a polarizationisotropic cavity composed of two plane parallel mirrors. The complex (slowly varying) amplitude of the vector electric field averaged over the longitudinal coordinate of the laser cavity is presented as a superposition of two orthogonally polarized components:

$$
\vec{E}(x, y, t)=\vec{e}_{1} E_{1}(x, y, t)+\vec{e}_{2} E_{2}(x, y, t),
$$

where $\vec{e}_{1}$ and $\vec{e}_{2}$ are the unit vectors in the linear polarization basis $\vec{e}_{1}=\vec{e}_{x}$ and $\vec{e}_{2}=\vec{e}_{y}$ or, alternatively, in the circular basis $\vec{e}_{1}=\vec{e}_{+}$and $\vec{e}_{2}=\vec{e}_{-}$. The relaxation times of the population inversion and polarization of the gain medium (the transverse relaxation) are assumed to be negligibly small if compared with the lifetime of intracavity light-field relaxation (a so-called class-A laser).

To calculate the evolution of transverse structure of the complex-valued electric vector field formed inside the laser cavity with the isotropic saturable gain $G(I)$, we use the following system of extended Ginsburg-Landau equations:

$$
\frac{\partial E_{1,2}}{\partial t}=\left(d_{\mathrm{Re}}+i d_{\mathrm{Im}}\right) \nabla_{\perp}^{2} E_{1,2}+[G(I)-\eta] E_{1,2} .
$$

Here $d_{\operatorname{Re}}$ and $d_{\operatorname{Im}}$ are respectively the diffusion and diffraction coefficients, $\nabla_{\perp}^{2}=\partial^{2} / x^{2}+\partial^{2} / y^{2}$ denotes the transverse Laplacian, the transverse coordinates $x$ and $y$ are scaled to the Fresnel zone width $(\sqrt{\lambda L / 4 \pi(1-R)}$, with $R$ being the product of reflectivity coefficients of the cavity mirrors [7]), $I=\left|E_{1}\right|^{2}+\left|E_{2}\right|^{2}$ is the total intracavity light intensity normalized to the saturated intensity of the gain medium, $G(I)=g_{0} /(1+I)$ the saturable gain (with $g_{0}$ being the unsaturated gain), and $\eta$ the linear intracavity losses. Eq. (2) describes the interaction between the fields $E_{1}(x, y, t)$ and $E_{2}(x, y, t)$ via the effect of cross-saturation of the gain medium. The gain medium is spatially homogeneous and isotropic. This implies a uniform angular distribution of the dipole momenta of active centres, which takes place, e.g., in glasses [8]. For simplicity, we assume that the coefficients of self- and cross-saturation are equal to each other.

To find spatially homogeneous and steady-state solutions of Eq. (2), we set the temporal and spatial derivatives equal to zero. This yields in the equation $E_{1} E_{1}^{*}+E_{2} E_{2}^{*}=g_{0} / \eta-1$ for the total intracavity light intensity, which implies polarization multistability. In other words, when the unsaturated gain is above the threshold $\left(g_{0}>\eta\right)$, the polarization state of the laser emission can take any value because the intensities of the orthogonal polarization components, $I_{1}=\left|E_{1}\right|^{2}$ and $I_{2}=\left|E_{2}\right|^{2}$, vary arbitrarily in the range from 0 to $g_{0} / \eta-1$, in accordance with the relation 
$I_{2}=\sqrt{g_{0} / \eta-1-I_{1}}$. Moreover, then the phase difference between the orthogonal polarization components can also take arbitrary values, since Eq. (2) is invariant under phase shifting.

\section{Numerical simulations}

In order to obtain the laser-field patterns for the total intensity/polarization and for the intensity/phase of each orthogonal component, we have integrated Eq. (2) numerically using a split-step Fourier-transform method. The boundary conditions have been defined at the edge of the circular diaphragm which is modelled by the $\eta$ parameter in the form of super-Gaussian function of the radius $r$. The initial conditions have been specified as a random noise or as some injected optical field. These conditions are applied to Eq. (2) for a short time to trigger formation of laserfield patterns at some fixed $N_{F}$. The parameters of the injected optical fields such as their shape, size and polarization, have been changed for each individual case.

The numerical analysis of Eq. (2) has been carried out in the diaphragm-width range corresponding to moderate Fresnel numbers $\left(1<N_{F}<10\right)$, where the regular RFP-beam structures are revealed that manifest axially symmetric total intensity profiles with non-uniform distributions of the polarization states. Note that the laser field has a polarization-bistable $\mathrm{TEM}_{00}$-mode structure [9] at small laser-cavity Fresnel numbers $\left(N_{F} \cong 1\right.$ ). On the contrary, we deal with irregular structures, with polarization topological defects present [10], whenever the cavity Fresnel numbers become larger $\left(N_{F}>>1\right)$.

\section{Results and discussion}

We have found five different types of the RFP-mode structures with distinct polarization state distributions, which depend on both the laser-cavity Fresnel number and the initial conditions. The structures are composed of two orthogonally polarized transverse modes, at least one of which containing a spiral phase defect (a vortex). Due to vector interaction via the mechanism of crosssaturation of the gain medium, the orthogonal components take the forms of complementary structures, and their phases evolve in a consistent manner.

Fig. 1a and Fig. 1b show the type I RFP-mode structures formed at $N_{F} \cong 3$ for the linear and circular bases, respectively. They are composed of two Laguerre-Gauss modes. These are the fundamental mode $\mathrm{TEM}_{00}$ (Fig. 1d, f) and the first-order azimuthal mode $\mathrm{TEM}_{01}$ * with the spiral
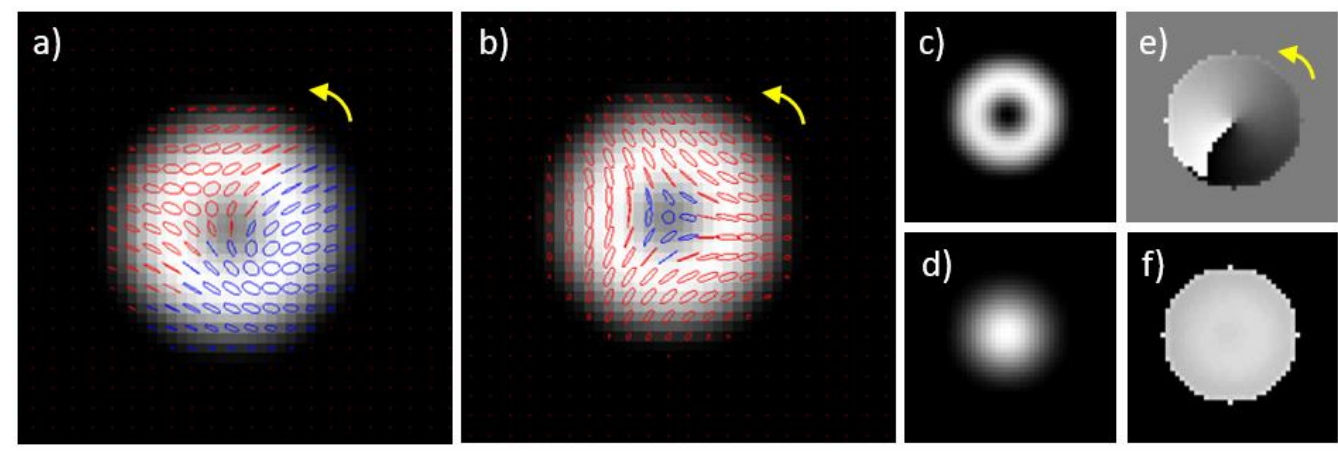

Fig. 1. Type I RFP-mode structures calculated at $N_{F} \cong 3$ : transverse distributions of total intensity and polarization ellipses for the cases $\vec{e}_{1}=\vec{e}_{x}, \vec{e}_{2}=\vec{e}_{y}$ (a) and $\vec{e}_{1}=\vec{e}_{+}, \vec{e}_{2}=\vec{e}_{-}$(b), instant intensity (c)/(d) and phase (e)/(f) for the first/second polarization component. Red and blue ellipses denote respectively left-handed and right-handed polarization states. Arrows indicate rotation directions for the polarization structure and the phase.

Ukr. J. Phys. Opt. 2017, Volume 18, Issue 1 
phase structure (Fig. 1c, e). Vector combining of these modes produces instant polarization structures of the first-order full Poincare beams, which is very much the same as the processes studied in Ref. [2]. However, a fundamental difference from Ref. [2] is that the RFP-structure is formed inside the laser cavity. Therefore, the orthogonally polarized Laguerre-Gauss modes of different orders have different Gouy phase shifts per round trip.

Our calculations have testified that the frequency of the spiral phase circulation for the $\mathrm{TEM}_{01} *$ mode is about 2.5 times higher than the frequency of the radial phase change for the $\mathrm{TEM}_{00}$ mode. Coupling of such transverse modes of different frequencies, which results in a linear temporal change of the phase difference, leads to periodic rotation of the polarization structures at a constant angular velocity. In this case the total polarization structure and the phase structure of the vortex component are rotating in the same direction, although at different rates (see Fig. 1).

As seen from Fig. 2a, b, the second-order RFP-modes appear for larger cavity Fresnel numbers $\left(N_{F} \geq 5\right)$. They are composed of the first-order azimuthal mode $\mathrm{TEM}_{01 *}$ (see Fig. 2c, e) and the first-order radial mode $\mathrm{TEM}_{10}$ (see Fig. 2d, f). In this case the frequency of the phase circulation of the $\mathrm{TEM}_{01 *}$ mode becomes about two times less than the frequency of the radial phase change for the $\mathrm{TEM}_{10}$ mode. That is why the polarization structure of the type II RFP-mode and the phase structure of its vortex component rotate in the opposite directions (see Fig. 2).
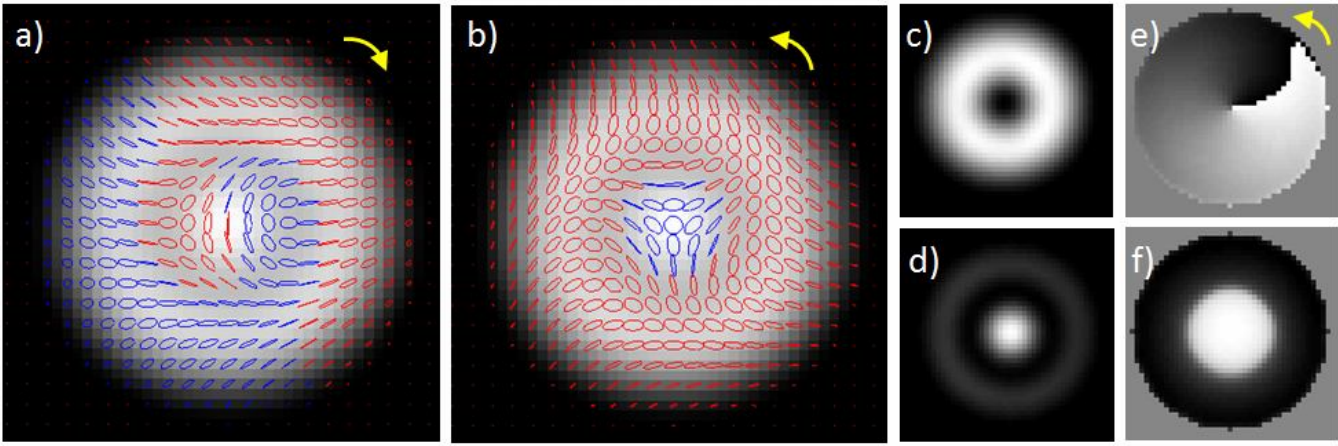

Fig. 2. Type II RFP-mode structures calculated at $N_{F} \cong 6$. Notation is the same as in Fig. 1 .

All the RFP-mode structures contain polarization topological defects. These are the lines of linear polarization states (the L-lines) which separate the domains of different handednesses (see Fig. 1a, b and Fig. 2a, b) and the points of right-handed $(\mathrm{C}+)$ and left-handed $(\mathrm{C}-)$ circular polarizations. The RFP-structures of the types I and II for the case $\vec{e}_{1}=\vec{e}_{\mathrm{x}}$ and $\vec{e}_{2}=\vec{e}_{\mathrm{y}}$ (see Fig. 1a and Fig. 2a) contain a pair of the $\mathrm{C}$ point defects: the $\mathrm{C}+$ defect surrounded by a pattern of ellipses having a 'lemon' morphology, with a single radial direction of the ellipses' major axes, and the Cdefect having a 'star' morphology, with three radial directions of the ellipses' major axes [11]. As seen from Fig. 3, the radial position of the $\mathrm{C}$ point defects corresponds to intersection of the intensity profile curves for the orthogonal polarization components 1 and 2 . Their azimuthal orientation is given by the phase difference between the orthogonal components, which varies in time. For the case $\vec{e}_{1}=\vec{e}_{+}$and $\vec{e}_{2}=\vec{e}_{-}$, the RFP-structures include only the $\mathrm{C}+$ defects, which are located at the beam centre and manifest the star morphology (see Fig. 1b and Fig. 2b). Change in the sign of the topological charge of the $\mathrm{TEM}_{01 *}$ component leads to the corresponding change in the morphology of the $\mathrm{C}$ points of the RFP-modes (the lemon pattern of the ellipses transforms into the star structure, and vice versa) and to the change in their rotation direction. 

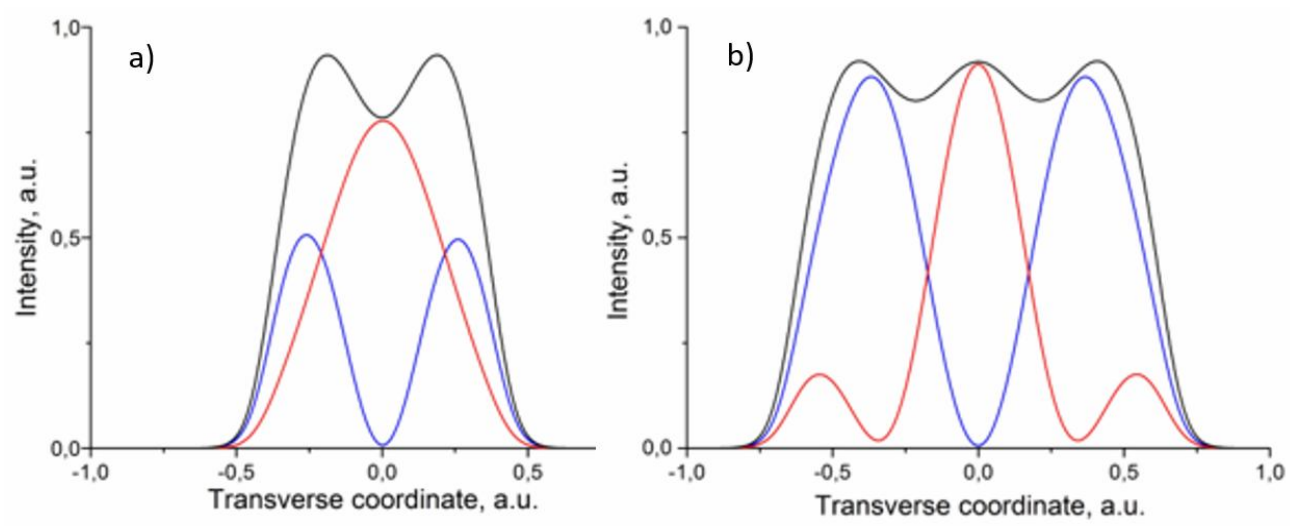

Fig. 3. Intensity profiles for the orthogonal polarization components and the resulting fields calculated for the RFP-mode structures of the types I (a) and II (b): blue and red lines correspond to the components 1 and 2 , and black line to the total intensity.

As seen from Figs. 4-7, the RFP-mode structures of the types III, IV and V represent bound states of the transverse modes which, in contrast to the structures of the types I and II, have no scalar counterparts. Their distinguishing feature is that each of their orthogonal components contains the same number of vortices, all having the same sign. In this case the frequencies of the orthogonally polarized transverse modes are the same, because their Gouy phase shifts per round trip are identical. However, since the vortices are in a coupled state, they force the orbital motion of the intensity structures for each polarization component, due to a presence of azimuthal phasegradient structure in the vicinity of the nodal point. Notice that similar rotational motion of a scalar cluster of vortices forced by local azimuthal phase gradients has been observed experimentally for the laser-like photorefractive oscillator operating at a fixed polarization $[12,13]$.

The type III RFP-structures can be created roughly in the same range of $N_{F}$ as the type I structures, and they look similar. However, there is a significant difference. The orthogonal components of the type III structures have the identical crescent shapes (Fig. 4c, d), they rotate with the same speed, and their phase difference, $\Delta \varphi$, can take arbitrary values. The type III RFPstructure under the conditions $\vec{e}_{1}=\vec{e}_{\mathrm{x}}, \vec{e}_{2}=\vec{e}_{\mathrm{y}}$ and $\Delta \varphi=0$ (see Fig. 4a) has the same polarization configuration (in both the polarization defect locations and their morphologies) and the same rotation frequency as the type I structure for $\vec{e}_{1}=\vec{e}_{x}$ and $\vec{e}_{2}=\vec{e}_{y}$ (see Fig. 1a). On the other hand, the
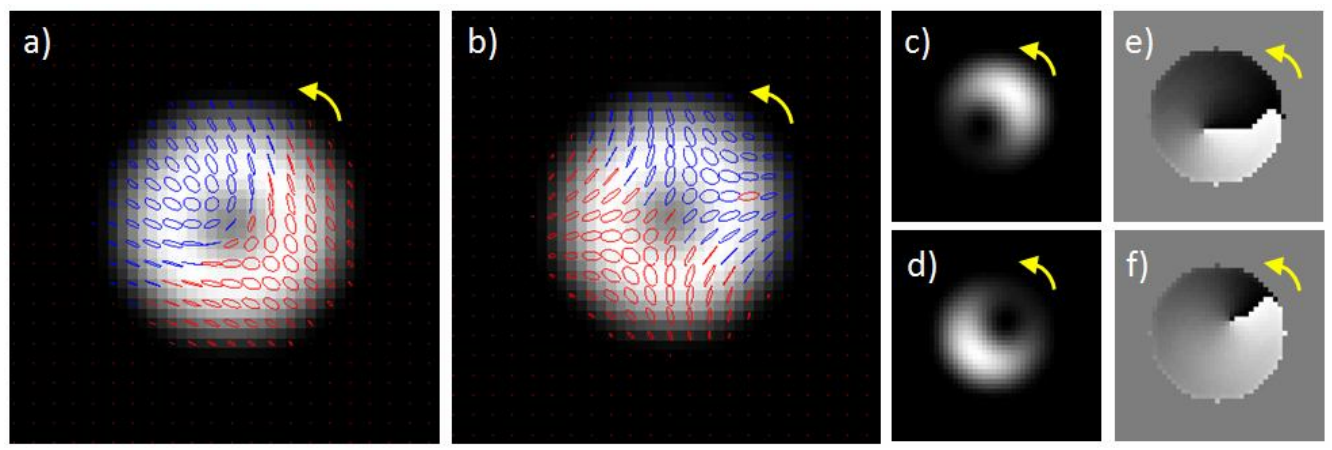

Fig. 4. Type III RFP-mode structures calculated at $N_{F} \cong 3, \Delta \varphi=0$. Notation is the same as in Fig. 1 . 
type III RFP-structure for $\vec{e}_{1}=\vec{e}_{x}, \vec{e}_{2}=\vec{e}_{y}$ and $\Delta \varphi=\pi / 2$ (Fig. 5a) reveals similarity with the type I RFP-structure for $\vec{e}_{1}=\vec{e}_{+}, \vec{e}_{2}=\vec{e}_{-}$: the both structures have central locations of the $\mathrm{C}+$ defect and a round shape of the L-line. The differences between these structures are such that the $\mathrm{C}+$ defect in Fig. 1b has the star morphology, the defect displayed in Fig. 5a has the lemon morphology, and they rotate in the opposite directions.
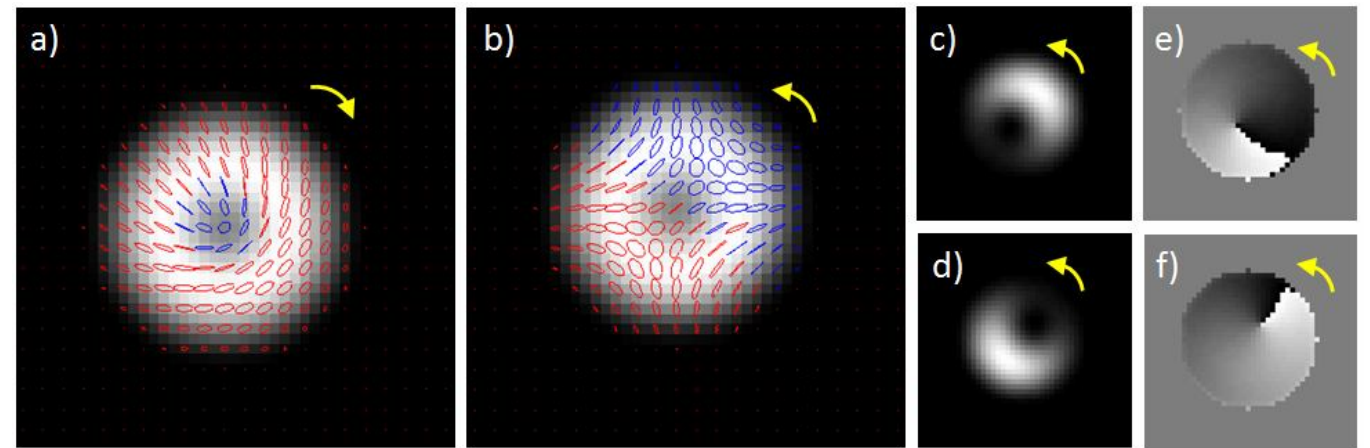

Fig. 5. Type III RFP-mode structures calculated at $N_{F} \cong 3, \Delta \varphi=\pi / 2$. Notation is the same as in Fig. 1 .
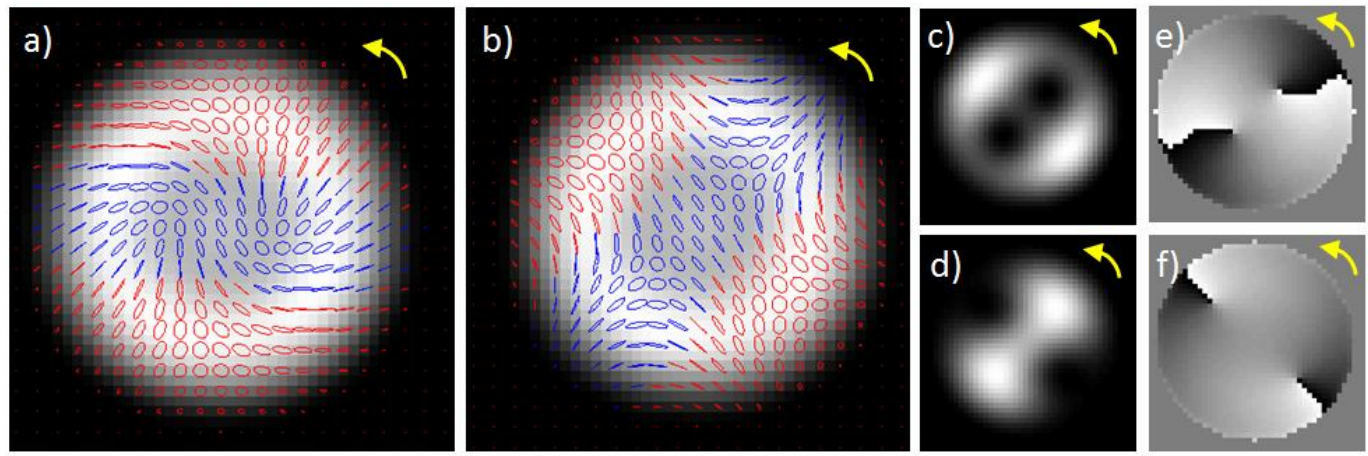

Fig. 6. Type IV RFP-mode structures calculated at $N_{F} \cong 6$. Notation is the same as in Fig. 1 .
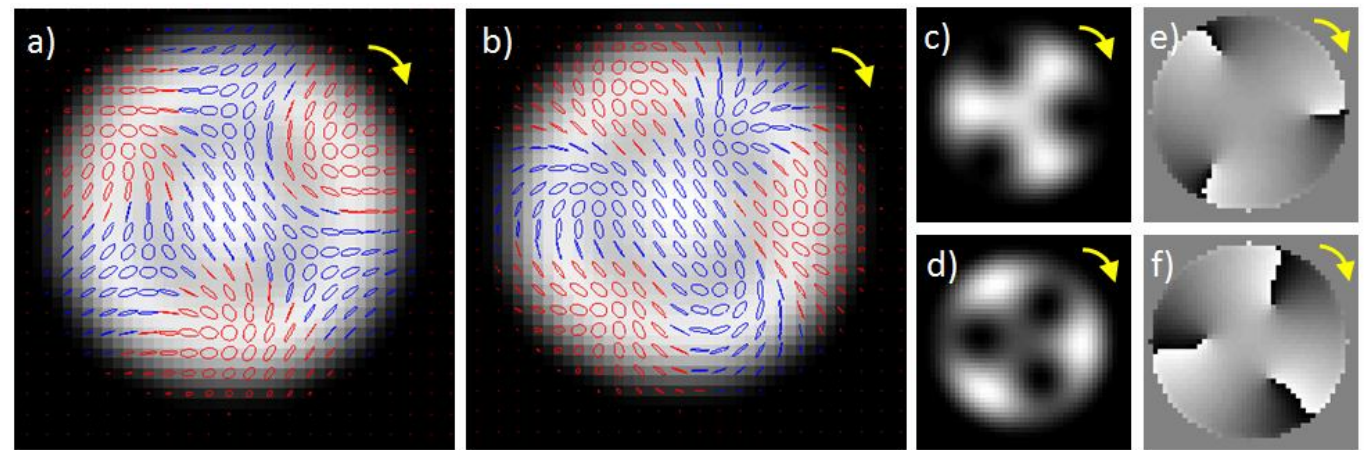

Fig. 7. Type V RFP-mode structures calculated at $N_{F} \cong 6$. Notation is the same as in Fig. 1 .

Each orthogonal component of the type IV RFP-modes contains a pair of vortices with the same signs (see Fig. 6c, d), which circulate at the same frequency. The total polarization pattern has the same circulating frequency (see Fig. 6a, b). The type IV structures contain two $\mathrm{C}+$ defects and two $\mathrm{C}$ - defects, respectively with the lemon and star morphologies for the linear polarization 
basis. For the circular-basis structure (see Fig. 6b), the both $\mathrm{C}+$ and $\mathrm{C}-$ defects reveal the star arrangement. Finally, the type $\mathrm{V}$ structures shown in Fig. 7a, b have even more complicated polarization structures. They contain three $\mathrm{C}+$ defects and three $\mathrm{C}-$ defects. The phase point defects also have the same signs.

\section{Conclusion}

In conclusion, we have studied numerically the RFP-modes formed in the isotropic laser with moderate Fresnel numbers. The modes reveal axially symmetric total-intensity profiles and inhomogeneous polarization patterns that include polarization topological defects rotating around the beam axis with constant velocities. We have found five different types of the RFP-modes, which represent 'composites' of mutually trapped and orthogonally polarized transverse structures. These are the pairs of Laguerre-Gauss modes of different orders ( $\mathrm{TEM}_{00}$ and $\mathrm{TEM}_{01}$, as well as $\mathrm{TEM}_{10}$ and $\mathrm{TEM}_{01^{*}}$ ) and the pairs of modes with complementary structures that exist only in the bound state. We have also demonstrated that the RFP-polarization structures in the linear polarization basis are quite different from those associated with the circular basis. The direction of rotation of the polarization structure is governed by the sign of the frequency difference between the orthogonally polarized fields, as well as by the sign of topological charge of the vortices available in the field structure.

\section{References}

1. Zhan Q, 2009. Cylindrical vector beams: from mathematical concepts to applications. Adv. Opt. Photon. 1: 1-57.

2. Beckley A, Brown T and Alonso M, 2010. Full Poincaré beams. Opt. Express. 18: 1077710785.

3. Wang L-G, 2012. Optical forces on submicron particles induced by full Poincaré beams. Opt. Express. 20: 20814-20826.

4. Milione G, Sztul H, Nolan D and Alfano R, 2011. Higher-order Poincare' sphere, Stokes parameters, and the angular momentum of light. Phys. Rev. Lett. 107: 053601/1-4.

5. Naidoo D, Roux F, Dudley A, Litvin I, Piccirillo B, Marrucci L and Forbes A, 2016. Controlled generation of higher-order Poincaré sphere beams from a laser. Nature Photon. 10: $327-332$.

6. Galvez E, Khadka S, Schubert W and Nomoto S, 2012. Poincaré-beam patterns produced by nonseparable superpositions of Laguerre-Gauss and polarization modes of light. Appl. Opt. 51: 2925-2934.

7. Rosanov N, Fedorov S and Shatsev A, 2006. Motion of clusters of weakly coupled twodimensional cavity solitons. JETP. 102: 547-555.

8. Khanin Ya. Principles of laser dynamics. Amsterdam, North-Holland/Elsevier (1995).

9. Zolotoverkh L and Lariontsev E, 2004. Bistability and chaos in an autonomous Nd: YAG laser with a weakly anisotropic resonator. Quant. Electron. 34: 727-730.

10. Gil L, 1993. Vector order parameter for an unpolarized laser and its vectorial topological defects. Phys. Rev. Lett. 70: 162-165.

11. Nye J. Natural focusing and fine structure of light: Caustics and wave dislocations. Bristol: IOP Publishing (1999).

12. Vaupel M and Weiss C, 1995. Circling optical vortices. Phys. Rev. A. 51: 4078-4085. 
13. Weiss C, Vaupel M, Staliunas K, Slekys G and Taranenko V, 1999. Vortices and solitons in lasers. Appl. Phys. B. 68: 151-168.

Krasnoshchekov Ye. A., Yaparov V. V. and Taranenko V. B. 2017. Rotating full Poincaré beams. Ukr.J.Phys.Opt. 18: 1 - 8.

Анотація. На основі чисельних розрахунків продемонстровано можливість появи повних променів Пуанкаре. Вони генеруються спонтанно або завдяки введенню оптичного поля в ізотропний лазер, який не містить селективних елементів, окрім циркулярної діафрагми, щчо контролюе число Френеля резонатора. Розраховано динамічні властивості циих променів. Для помірних чисел Френеля виявлено п'ять різних типів повних лазерних мод Пуанкаре. Кожсна з них має аксіально-симетричний профіль і особливий розподіл поляризаиї̈, який повертається навколо осі променя з постійною швидкістю. 